\title{
MULTI Phase M/G/1 Queue with Bernoulli Feedback and Multiple Server Vacation
}

\author{
S. Maragatha Sundari \\ Research scholar,Sathyabama university,chennai \\ Assoc.Prof,Dept of Mathematics,RIT,Chennai
}

\author{
S.Srinivasan \\ Prof. \& HOD, Dept.of Mathematics \\ BSA University, Chennai.
}

\begin{abstract}
In this paper, a multi phase M/G/1queueing system with Bernoulli feedback where the server takes multiple vacation is considered. All the poisson arrivals with mean arrival rate will demand any of the multi essential services.. The service times of the first essential service are assumed to follow a general distribution $\mathrm{B}_{\mathrm{i}}(\mathrm{v})$. After the completion of any of the $\mathrm{n}$ services, if the customer is dissatisfied he can join the tail of the queue for receiving another regular service with probability $\mathrm{p}$. Otherwise the customer may depart from the system with the probability q=1-p. If there is no customer in the queue, then the server can go for vacation and vacation periods are exponentially distributed with mean vacation time $\frac{1}{r}$.On returning from vacation, if the server again founds no customer waiting in the queue, then it again goes for vacation. The server continues to go for vacation until he finds at least one customer in the system. We find the time dependent probability generating function in terms of Laplace transforms and derive explicitly the corresponding steady state results.
\end{abstract}

\section{Mathematics Subjects Classification: 60K25, 62K30.}

Keywords- steady state solution, transient solution, Bernoulli feedback queue, multiple vacation.

\section{INTRODUCTION}

A multi phase M/G/1 Bernoulli feed back queue with multiple vacation is considered in this paper. In multiple vacation period the server keeps on taking vacation until there is at least • one customer present in the system.

Many authors have taken considerable efforts to study about Bernoulli feedback queueing systems Takacs was the first to study such a model, where the customers who completed their services feedback instantaneously to the tail of the queue with probability $\mathrm{p}$ or leaves the system forever with probability $\mathrm{q}=1$ - p. Many results with Bernoulli feedback are found [1 6].Several contributions have been made by dealing queueing systems of M/G/1 type.[7,9-15].Much research [8, 16 - 20]has studied queueing models under vacations. For complete reference on vacation models, one may refer to Doshi [17]and Tagagi [3].M/G/1 vacation models under the various service disciplines have been investigated[8,18,21-24].

The rest of the article is as follows.The model under • consideration is described in section 2.In section 3 we analyze
The model by giving the definitions and equations governing the system.Using these equations the probability generating function of queue length are obtained in section 4.In section 5 ,the steady state solution is obtained. Some operating characteristics are obtained in section 6.In section 7,the mean waiting time is derived.

\section{MATHEMATICAL DESCRIPTION OF THE MODEL}

We assume the following to describe the queueing model of our study.

- Customers arrive at the system one by one according to a Poisson stream with arrival rate $\lambda(>0)$.

- There is a single server which provides the first essential service to all arriving customers. Let $\mathrm{B}_{\mathrm{i}}(\mathrm{v})$ and $\mathrm{b}_{\mathrm{i}}(\mathrm{v})$ be the distribution and the density function of the first service times.

- As soon as the first service of a customer is complete, then with probability $r$ he may immediately enter into the second service or third service and so on upto $\mathrm{n}$ services or else with probability 1-r he may leave the system.

- After completion of service, if the customer is dissatisfied with any of the $\mathrm{n}$ services, he can immediately join the tail of the original queue as a feedback customer for receiving another regular service with probability $\mathrm{p}$. otherwise the customer may depart forever from the system with probability $q=1-p$.

- The customer both newly arrived and those that are fed aback are served in the order in which they join the tail of the original queue.

Service times are assumed to be general with the distribution function $B_{i}(v)$ respectively and the density function $b_{i}(v)$, respectively.i= 1 to $\mathrm{n}$.

Further, $s_{i}(x) d x$ is the probability of completion of the $i$-th type service given with that elapsed time is $\mathrm{x}$, so that

$$
\mathrm{s}_{\mathrm{i}}(\mathrm{x})=\frac{b_{i}(x)}{1-B_{i}(x)}, \mathrm{i}=1,2,3 \ldots . \mathrm{n}
$$

and therefore,

$$
b_{i}(v)=s_{i}(v) \exp \left(-\int_{0}^{x} s_{i}(x) d x\right) \quad i=1,2,3 \ldots \ldots
$$

If there is no customer waiting in the queue then the server goes for a vacation

On returning from vacation if the server again founds no customer waiting in the queue then it goes for another vacation. So the server takes multiple vacation. The vacation periods are 
exponentially distributed with mean vacation time $\frac{1}{r}$. The customer are served according to the first come, first served rule.

\section{EQUATIONS GOVERNING THE SYSTEM}

We define,

$\mathrm{P}_{\mathrm{n}}^{(1)}(\mathrm{x}, \mathrm{t})=$ Probability that at time $\mathrm{t}$, that are $\mathrm{n}(\geq 0)$ customers in the queue excluding one customer in the first type of service and the elapsed service time for this customer is $\mathrm{x}$.

$$
\begin{aligned}
& P_{i}^{(j)}(t)=\int_{0}^{\infty} P_{i}^{(j)}(x, t) d x \quad j= \\
& 1,2 \ldots \ldots n
\end{aligned}
$$

Denotes the Probability that at time $\mathrm{t}$ there are $i$ customers in the queue excluding the one customer being provided the $j^{\text {th }}$ optional service irrespective of the value of $x$.

$\mathrm{V}_{\mathrm{n}}(\mathrm{t})=$ Probability that at time $\mathrm{t}$, there are $\mathrm{n}(\geq 0)$ customers in the queue and the server is on vacation $\frac{\partial}{\partial x} P_{i}^{(j)}(x, t)+\frac{\partial}{\partial x} P_{i}^{(j)}(x, t)+\left[\lambda+s_{i}(x)\right] P_{i}^{(j)}(x, t)=$ $\lambda P_{i-1}^{(j)}(\mathrm{x}, \mathrm{t}), i=1,2 \ldots \ldots \ldots, j=1,2 \ldots \ldots . . n$

$\frac{\partial}{\partial x} P_{0}^{(j)}(x, t)+\frac{\partial}{\partial x} P_{0}^{(j)}(x, t)+\left[\lambda+s_{j}(x)\right] P_{0}^{(j)}(x, t)=0$

$\frac{d}{d t} \mathrm{~V}_{0}(\mathrm{t})=-(\lambda+\gamma) \mathrm{V}_{0}(\mathrm{t})+(1-\mathrm{r}) \mathrm{q} \int_{0}^{\infty} \mathrm{P}_{0}^{(1)}(\mathrm{x}, \mathrm{t}) \mathrm{s}_{1}(\mathrm{x}) \mathrm{dx}$

$+\sum_{j=2}^{n}\left[q \int_{0}^{\infty} P_{0}^{(j)}(x, t) s_{j}(x) d x\right] \gamma V_{0}(t)$

$\frac{d}{d t} \mathrm{~V}_{\mathrm{n}}(\mathrm{t})=-(\lambda+\gamma) \mathrm{V}_{\mathrm{n}}(\mathrm{t})+\lambda \mathrm{V}_{\mathrm{n}-1}(\mathrm{t})$

Equations (4-6) are to be solved subject to the following boundary conditions:-

$$
\begin{aligned}
\mathrm{P}_{0}^{(1)}(0, \mathrm{t})= & (1-\mathrm{r}) \mathrm{p} \int_{0}^{x} \mathrm{P}_{0}^{(1)}(\mathrm{x}, \mathrm{t}) \mathrm{s}_{1}(\mathrm{x}) \mathrm{dx} \\
& +\sum_{j=2}^{n} P \int_{0}^{\infty} P_{0}^{(j)}(x, t) s_{j}(x) d x \\
& +(1-r) q \int_{0}^{\infty} P_{1}^{(1)}(x, t) s_{1}(x) d x \\
& +\sum_{j=2}^{n} P \int_{0}^{\infty} P_{1}^{(j)}(x, t) s_{j}(x) d x . j=2,3 \ldots . . .
\end{aligned}
$$

$$
\begin{aligned}
\mathrm{P}_{\mathrm{i}}^{(1)}(0, \mathrm{t})= & (1-\mathrm{r}) \mathrm{p} \int_{0}^{\infty} \mathrm{P}_{\mathrm{n}}^{(1)}(\mathrm{x}, \mathrm{t}) \mathrm{s}_{1}(\mathrm{x}) \mathrm{dx} \\
& +\sum_{j=2}^{n} P \int_{0}^{\infty} P_{i}^{(j)}(x, t) s_{j}(x) d x \\
& +(1-\mathrm{r}) \mathrm{q} \int_{0}^{\infty} \mathrm{P}_{\mathrm{n}+1}{ }^{(1)}(\mathrm{x}, \mathrm{t}) \mathrm{s}_{1}(\mathrm{x}) \mathrm{dx} \\
& +\sum_{j=2}^{n} q \int_{0}^{\infty} P_{i+1}^{(j)}(x, t) s_{j}(x) \quad j=2,3 \ldots . . n
\end{aligned}
$$

In general,
$P_{i}^{(j)}(0, t)=r \int_{0}^{\infty} P_{i}^{(1)}(r, t) s_{1}(x) d x j=2$ to $n$

(9)

We assume that initially there is no customer in the system, the server is not under vacation and the server is idle. So the inital conditions are $\mathrm{V}_{0}(0)=1, \mathrm{~V}_{\mathrm{n}}(0)=0, \quad \mathrm{P}_{\mathrm{i}}^{(\mathrm{j})}(0)=0$ for $\mathrm{n}=0,1,2$. , $\mathrm{j}=1,2,3 \ldots \ldots . . \mathrm{n}$

\section{TIME DEPENDENT SOLUTION}

For the above set of differential difference equations, the tnansient solution is to be obtained in this section. We define the probability generating functions as,

$\mathrm{P}_{\mathrm{q}}^{(\mathrm{j})}(\mathrm{x}, \mathrm{z}, \mathrm{t})=\sum_{i=0}^{\infty} \mathrm{Z}^{i} \mathrm{P}_{i}^{\left({ }^{(j)}\right.}(\mathrm{x}, \mathrm{t}), \mathrm{P}_{\mathrm{q}}^{(\mathrm{j})}(\mathrm{Z}, \mathrm{t})=\sum_{n=0}^{\infty} \mathrm{Z}^{\mathrm{i}} \mathrm{P}_{\mathrm{i}}^{(\mathrm{j})}(\mathrm{t})$, $j=1$ to $n$

$\mathrm{P}_{\mathrm{q}}^{(3)}(\mathrm{x}, \mathrm{z}, \mathrm{t})=\sum_{n=0}^{\infty} \mathrm{Z}^{\mathrm{n}} \mathrm{P}_{\mathrm{n}}{ }^{(3)}(\mathrm{x}, \mathrm{t}), \mathrm{P}_{\mathrm{q}}^{(3)}(\mathrm{Z}, \mathrm{t})=\sum_{n=0}^{\infty} \mathrm{Z}^{\mathrm{n}} \mathrm{P}_{\mathrm{n}}{ }^{(3)}$

(t)

$\mathrm{V}(\mathrm{Z}, \mathrm{t})=\sum_{n=0}^{\infty} \mathrm{Z}^{\mathrm{n}} \mathrm{V}_{\mathrm{n}}(\mathrm{t})$

Define the Laplace transform of $\mathrm{f}(\mathrm{t})$ as $\bar{f}(\mathrm{~s})=\int_{0}^{\infty} \mathrm{e}^{-\mathrm{st}} \mathrm{f}(\mathrm{t}) \mathrm{dt}$, $\mathrm{R}(\mathrm{s})>0$

Taking Laplace transforms of equations (3) - (9) and using (10) we have,

$\frac{\partial}{\partial x} \bar{p}_{i}^{(j)}(\mathrm{x}, \mathrm{s})+\left(\mathrm{s}+\lambda+\mathrm{s}_{\mathrm{j}}(\mathrm{x})\right) \bar{p}_{i}^{(j)}(\mathrm{x}, \mathrm{s})=\lambda \bar{p}_{i-1}{ }^{(j)}(\mathrm{x}, \mathrm{s})$,

$i=1,2 . . j=1,2 \ldots . n$

$\frac{\partial}{\partial x} \bar{p}_{0}^{(j)}(\mathrm{x}, \mathrm{s})+\left(\mathrm{s}+\lambda+\mathrm{s}_{1}(\mathrm{x})\right) \bar{p}_{0}^{(j)}(\mathrm{x}, \mathrm{s})=0 \quad j=1,2 \ldots . n$

$(\mathrm{s}+\lambda+\Upsilon) \overline{V_{0}}(\mathrm{~s})=1+(1-\mathrm{r}) \mathrm{q} \int_{0}^{\infty} \bar{p}_{0}^{(1)}(\mathrm{x}, \mathrm{s}) \mathrm{s}_{1}(\mathrm{x}) \mathrm{dx}$

$+\sum_{j=2}^{n}\left[\mathrm{q} \int_{0}^{\infty} \bar{p}_{0}^{(j)}(\mathrm{x}, \mathrm{s}) \mathrm{s}_{\mathrm{j}}(\mathrm{x}) \mathrm{dx}\right]$

$(\mathrm{s}+\lambda+\Upsilon) \overline{V_{n}}(\mathrm{~s})=\lambda \overline{V_{n-1}}(\mathrm{~s}) \mathrm{n}=1,2 \ldots$

$\bar{p}_{0}^{(1)}(0, \mathrm{~s})=(1-\mathrm{r}) \mathrm{p} \int_{0}^{\infty} \bar{p}_{0}^{(1)}(\mathrm{x}, \mathrm{s}) \mathrm{s}_{1}(\mathrm{x}) \mathrm{dx}$

$+\sum_{j=2}^{n}\left[\mathrm{P} \int_{0}^{\infty} \bar{p}_{0}^{(j)}(\mathrm{x}, \mathrm{s}) \mathrm{s}_{\mathrm{j}}(\mathrm{x}) \mathrm{dx}\right]$ 


$$
\begin{aligned}
& +(1-\mathrm{r}) \mathrm{q} \int_{0}^{\infty} \bar{p}_{1}^{(1)}(\mathrm{x}, \mathrm{s}) \mathrm{s}_{1}(\mathrm{x}) \mathrm{dx} \\
& +\sum_{j=2}^{n}\left[\mathrm{q} \int_{0}^{\infty} \bar{p}_{0}^{(j)}(\mathrm{x}, \mathrm{s}) \mathrm{s}_{\mathrm{j}}(\mathrm{x}) \mathrm{dx}\right. \\
& +\Upsilon V_{1}(s)
\end{aligned}
$$

$$
\bar{p}_{n}{ }^{(1)}(0, \mathrm{~s})=(1-\mathrm{r}) \mathrm{p} \int_{0}^{\infty} \bar{p}_{n}{ }^{(1)}(\mathrm{x}, \mathrm{s}) \mathrm{s}_{1}(\mathrm{x}) \mathrm{dx}
$$

$$
\begin{aligned}
& +\sum_{j=2}^{n} \mathrm{p} \int_{0}^{\infty} \bar{p}_{n}{ }^{(j)}(\mathrm{x}, \mathrm{s}) \mathrm{s}_{\mathrm{j}}(\mathrm{x}) \mathrm{dx} \\
& +(1-\mathrm{r}) \mathrm{q} \int_{0}^{\infty} \bar{p}_{n+1}{ }^{(1)}(\mathrm{x}, \mathrm{s}) \mathrm{s}_{1}(\mathrm{x}) \mathrm{dx} \\
& +\sum_{j=2}^{n} \mathrm{q} \int_{0}^{\infty} \bar{p}_{i+1}{ }^{(j)}(\mathrm{x}, \mathrm{s}) \mathrm{s}_{\mathrm{j}}(\mathrm{x}) \mathrm{dx} \\
& +\Upsilon \overline{V_{n+1}}(\mathrm{~s}) \quad i=1,2 \ldots . .
\end{aligned}
$$

In general,

$$
\bar{p}_{i}^{(j)}(0, \mathrm{~s})=\mathrm{r} \int_{0}^{\infty} \bar{p}_{i}^{(1)}(\mathrm{x}, \mathrm{s}) \mathrm{s}_{1}(\mathrm{x}) \mathrm{dx} j=2 \text { to } n
$$

$i=1,2 \ldots$

Multiply equations $(15-20)$ by suitable powers of $z$, summing over $\mathrm{n} \&$ using the generating functions, we get

$$
\frac{\partial}{\partial x} \bar{p}_{q}{ }^{(j)}(\mathrm{x}, \mathrm{z}, \mathrm{s})+\left(s+\lambda-\lambda \mathrm{z}+\mathrm{s}_{\mathrm{j}}(\mathrm{x})\right) \bar{p}_{q}{ }^{(j)}(\mathrm{x}, \mathrm{z}, \mathrm{s})=0
$$

$j=1$ to $n$

$$
\begin{aligned}
& (s+\lambda+\Upsilon-\lambda \mathrm{z}) \bar{V}(\mathrm{z}, \mathrm{s})=1+(1-\mathrm{r}) \mathrm{q} \int_{0}^{\infty} \bar{p}_{0}^{(1)}(\mathrm{x}, \mathrm{s}) \mathrm{s}_{1}(\mathrm{x}) \mathrm{dx} \\
& +\sum_{j=2}^{n} \mathrm{q} \int_{0}^{\infty} \bar{p}_{0}^{(j)}(\mathrm{x}, \mathrm{s}) \mathrm{s}_{\mathrm{j}}(\mathrm{x}) \mathrm{dx}+\Upsilon \overline{V_{0}}(\mathrm{~s})
\end{aligned}
$$

Next multiply both sides of eq (18) by z \& multiply both sides of eq (19) by $\mathrm{Z}^{\mathrm{n}+1}$, Summing over $\mathrm{n}=1$ to $\infty$ and adding the two result $\&$ using probability generating funtion we get,

$\mathrm{Z} \bar{p}_{q}{ }^{(1)}(0, \mathrm{z}, \mathrm{s})=\bar{V}(\mathrm{z}, \mathrm{s})+(1-\mathrm{r}) \mathrm{q} \int_{0}^{\infty} \bar{p}_{q}{ }^{(1)}(\mathrm{x}, \mathrm{z}, \mathrm{s}) \mathrm{s}_{1}(\mathrm{x}) \mathrm{dx}$

$$
\begin{aligned}
& -(1-\mathrm{r}) \mathrm{q} \int_{0}^{\infty} \bar{p}_{0}{ }^{(1)}(\mathrm{x}, \mathrm{s}) \mathrm{s}_{1}(\mathrm{x}) \mathrm{d} \mathrm{x} \\
& +\sum_{j=2}^{n}\left[\mathrm{q} \int_{0}^{\infty} \bar{p}_{0}{ }^{(j)}(\mathrm{x}, \mathrm{z}, \mathrm{s}) \mathrm{s}_{\mathrm{j}}(\mathrm{x}) \mathrm{dx}\right] \\
& -\sum_{j=2}^{n}\left[\mathrm{q} \int_{0}^{\infty} \bar{p}_{0}^{(j)}(\mathrm{x}, \mathrm{s}) \mathrm{s}_{\mathrm{j}}(\mathrm{x}) \mathrm{dx}\right] \_(23)
\end{aligned}
$$

Similarly using the rule of multiplying with powers of $\mathrm{Z}$ to the equations (20), and using the probability generating funtions, we get

$$
\bar{p}_{q}{ }^{(j)}(\mathrm{o}, \mathrm{z}, \mathrm{s})=\mathrm{r} \int_{0}^{\infty} \bar{p}_{q}{ }^{(1)}(\mathrm{x}, \mathrm{z}, \mathrm{s}) \mathrm{s}_{1}(\mathrm{x}) \mathrm{dx}
$$

Integrating equation (21), we get

$$
\bar{p}_{q}{ }^{(j)}(\mathrm{x}, \mathrm{z}, \mathrm{s})=\bar{p}_{q}{ }^{(j)}(0, \mathrm{z}, \mathrm{s}) \exp \left[-(\mathrm{s}+\lambda-\lambda \mathrm{z}) \mathrm{x}-\int_{0}^{x} \mathrm{~s}(\mathrm{t}) \mathrm{dt}\right.
$$

$\bar{p}_{q}{ }^{(j)}(0, \mathrm{z}, \mathrm{s}), \mathrm{j}=1,2, \ldots \mathrm{n}$ are given by the equation (23) and (24) Integrating (21) with respect to $\mathrm{x}$, we get

$$
\bar{p}_{q}^{(j)}(\mathrm{z}, \mathrm{s})=\bar{p}_{q}{ }^{(j)}(0, \mathrm{z}, \mathrm{s})\left(\frac{1-\bar{K}_{j}(s+\lambda-\lambda z)}{s+\lambda-\lambda z}\right)
$$

Where $\bar{K}_{j}(\mathrm{~s}+\lambda-\lambda \mathrm{z})=\int_{0}^{\infty} \exp [-(\mathrm{s}+\lambda-\lambda \mathrm{z}) \mathrm{x}] \mathrm{dK}_{\mathrm{j}}(\mathrm{x})$

$j=1$ to $n$

From eq (22), we have

$$
\begin{aligned}
& -(1-\mathrm{r}) \mathrm{q} \int_{0}^{\infty} \bar{p}_{0}^{(1)}(\mathrm{x}, \mathrm{s}) \mathrm{s}_{1}(\mathrm{x}) \mathrm{dx} \\
& -\mathrm{q} \sum_{j=2}^{n}\left[\int_{0}^{\infty} \bar{p}_{0}^{(j)}(\mathrm{x}, \mathrm{s}) \mathrm{s}_{\mathrm{j}}(\mathrm{x}) \mathrm{dx}\right] \\
& =1+(-s-\lambda-\Upsilon+\lambda \mathrm{z}) \bar{V}(\mathrm{z}, \mathrm{s})+\Upsilon \overline{V_{0}}
\end{aligned}
$$

using the equations(26), (27) \& (28) and after simplifications we get 
$\mathrm{Z} \bar{p}_{q}{ }^{(j)}(0, \mathrm{z}, \mathrm{s})=(1-\mathrm{r}) \mathrm{q} \bar{p}_{q}{ }^{(1)}(0, \mathrm{z}, \mathrm{s}) \bar{K}_{1}(\mathrm{~s}+\lambda-\lambda \mathrm{z})$

$$
\begin{aligned}
& +\mathrm{q} \sum_{j=2}^{n} \int_{0}^{n} \bar{p}_{q}^{(j)}(0, \mathrm{z}, \mathrm{s}) \bar{K}_{j}(\mathrm{~s}+\lambda-\lambda \mathrm{z}) \\
& +(1-\mathrm{r}) \mathrm{pz} \bar{p}_{q}^{(1)}(0, \mathrm{z}, \mathrm{s}) \bar{K}_{1}(\mathrm{~s}+\lambda-\lambda \mathrm{z}) \\
& +\mathrm{pz} \sum_{j=2}^{n} \bar{p}_{q}{ }^{(1)}(0, \mathrm{z}, \mathrm{s}) \overline{\mathrm{K}}_{\mathrm{J}}(\mathrm{s}+\lambda-\lambda \mathrm{z}) \\
& +1+(-s-\lambda+\lambda \mathrm{z}) \bar{V}(\mathrm{z}, \mathrm{s})
\end{aligned}
$$

Substituting the values of $\bar{p}_{q}{ }^{(2)}(0, \mathrm{z}, \mathrm{s})$ and $\bar{p}_{q}{ }^{(3)}(0, \mathrm{z}, \mathrm{s})$, $Z \bar{P}_{q}{ }^{(1)}(0, \mathrm{z}, \mathrm{s})=(1-\mathrm{r}) \mathrm{q} \bar{P}_{q}{ }^{(1)}(0, \mathrm{~s}, \mathrm{z}) \overline{K_{1}}(s+\lambda-\lambda z)+$ $(1-r) p z \bar{P}_{q}{ }^{(1)}(0, \mathrm{z}, \mathrm{s}) \overline{K_{1}}(s+\lambda-\lambda z)+(\mathrm{q}+\mathrm{pz}) \overline{K_{2}}(s+\lambda-$ $\lambda z)\left[\mathrm{r} \bar{P}_{q}^{(1)}(0, \mathrm{z}, \mathrm{s}) \overline{K_{1}}(s+\lambda-\lambda z)\right](\mathrm{q}+\mathrm{pz}) \overline{K_{3}}(s+\lambda-$ $\lambda z)\left[r^{2} \bar{P}_{q}^{(1)}(0, \mathrm{z}, \mathrm{s}) \overline{K_{1}}(s+\lambda-\lambda z)\right.$

$\overline{K_{2}}(s+\lambda-\lambda z)+$ .$+\left((\mathrm{q}+\mathrm{pz}) r^{n-1} \prod_{j=1}^{n} \bar{K}_{J}(s+\lambda-\right.$

$\lambda z)+1+(-s-\lambda+\lambda \mathrm{z}) \bar{V}(\mathrm{z}, \mathrm{s})$

Hence ,

$$
\begin{aligned}
& \bar{p}_{q}^{(1)}(0, \mathrm{z}, \mathrm{s})=\frac{1+(-s-\lambda+\lambda \mathrm{z}) \bar{V}(\mathrm{z}, \mathrm{s})}{\operatorname{Dr}} \\
& \bar{p}_{q}{ }^{(j)}(0, \mathrm{z}, \mathrm{s})=\mathrm{r}^{\mathrm{j}-1} \bar{K}_{1}(\mathrm{~s}+\lambda-\lambda \mathrm{z})
\end{aligned}
$$

$$
\times \frac{1+(-s-\lambda+\lambda \mathrm{z}) \bar{V}(\mathrm{z}, \mathrm{s})}{\operatorname{Dr}} j=2 \text { to } n
$$

Where $\operatorname{Dr}=\mathrm{z}-(1-\mathrm{r}) \mathrm{q} \overline{K_{1}}(s+\lambda-\lambda z)-(1-\mathrm{r}) p z \overline{K_{1}}(s+\lambda-$ $\lambda z)-(q+p z)\left[\overline{K_{2}}(s+\lambda-\lambda z)\left[r \overline{K_{1}}(s+\lambda-\lambda z)\right]_{-}(q+\right.$ $p z)\left[\overline{K_{3}}(s+\lambda-\lambda z)\left[r^{2} \overline{K_{1}}(s+\lambda-\lambda z) \overline{K_{2}}(s+\lambda-\lambda z)\right]\right.$ $+(\mathrm{q}+\mathrm{pz}) r^{n-1} \prod_{j=1}^{n} \bar{K}_{J}(s+\lambda-\lambda z)$

From equations (26) and (27),

$\bar{p}_{q}^{(1)}(\mathrm{z}, \mathrm{s})=\frac{1+(-s-\lambda+\lambda \mathrm{z}) \bar{V}_{(\mathrm{z}, \mathrm{s})}}{\operatorname{Dr}} \quad\left(\frac{1-\bar{K}_{1}(s+\lambda-\lambda z)}{s+\lambda-\lambda z}\right)$ (34)

$\bar{p}_{q}{ }^{(j)}(\mathrm{z}, \mathrm{s})=r^{j-1} \overline{K_{1}}(s+\lambda-\lambda z) \frac{1+(-s-\lambda+\lambda \mathrm{z}) \bar{V}(\mathrm{z}, \mathrm{s})}{\operatorname{Dr}}$ $\left(\frac{1-\bar{K}_{j}(s+\lambda-\lambda z)}{s+\lambda-\lambda z}\right) j=2$ to $\mathrm{n}_{-}(35)$
Let $\bar{p}_{q}(\mathrm{z}, \mathrm{s})=\sum_{j=1}^{n} \bar{p}_{q}{ }^{(j)}(\mathrm{z}, \mathrm{s})$ denote the probability generating function of the number of queue irrespectiveof the type of service being provided.

$$
\bar{p}_{q} \quad(\mathrm{z}, \mathrm{s})=\frac{1+(-s-\lambda+\lambda \mathrm{z}) \bar{V}_{(\mathrm{z}, \mathrm{s})}}{\operatorname{Dr}}\left(\frac{N r}{s+\lambda-\lambda z}\right)
$$

Where

$$
\begin{aligned}
& \mathrm{Nr}=1-\overline{K_{1}}(s+\lambda-\lambda z) \\
& \quad+\sum_{j=2}^{n} r^{j-1} \overline{K_{1}}(s+\lambda-\lambda z)\left[1-\overline{K_{J}}(s+\lambda-\lambda z)\right] \_(37) \\
& \mathrm{Dr}=\mathrm{z}-(1-\mathrm{r}) \mathrm{q} \overline{K_{1}}(s+\lambda-\lambda z)-(1-r) p z \overline{K_{1}}(s+\lambda-\lambda z) \\
& -(q+p z) \overline{K_{2}}(s+\lambda-\lambda z)\left[r \overline{K_{1}}(s+\lambda-\lambda z)\right] \\
& -(q+p z) \overline{K_{3}}(s+\lambda-\lambda z)\left[r^{2} \overline{K_{1}}(s+\lambda-\lambda z) \overline{K_{2}}(s+\lambda-\lambda z)\right]
\end{aligned}
$$

If we let $\mathrm{z}=1$ in equation ( 36$)$, we can verify that $\bar{p}_{q} \quad(\mathrm{z}, \mathrm{s})+$ $\bar{V}(\mathrm{z}, \mathrm{s})=\frac{1}{\mathrm{~s}}$

Moreover, it can shown that the denominator of the right hand side of eq (36) has one zero inside the unit circle $|z|=1$, which is sufficient to determine the unknown $\bar{V}$ (z,s) appearing in the numerator.Therefore $\bar{p}_{q}$ (z,s), $\bar{p}_{q}{ }^{(j)}(\mathrm{z}, \mathrm{s}), j=1$ to $n$ are completely determined.

\section{THE STEADY STATE RESULTS}

To define the steady state probabilities leave the argument $t$ wherever it appears in the time dependent analysis. Then the corresponding steady state results can be obtained using Tauberian propert

$\lim _{s \rightarrow 0} s \bar{f}(\mathrm{~s})=\lim _{s \rightarrow \infty} f(t)$

Thus ,multiplying both sides of the equation (36) by s,taking limit as s tends to 0 ,applying the property and simplifying, we have $P_{q}(z)=V(z)\left[\frac{N(z)}{D(z)}\right]$

Where the numerator

$$
\begin{aligned}
& \mathrm{N}(\mathrm{z})=1-\overline{K_{1}}(\lambda-\lambda z)+\overline{K_{1}}(\lambda-\lambda z) \sum_{j=2}^{n} r^{j-1}\left[1-\overline{K_{J}}(\lambda-\lambda z)\right. \\
& D(z)=z-(1-r) q \overline{K_{1}}(\lambda-\lambda z)-(1-r) p z \overline{K_{1}} \quad(\lambda-\lambda z) \\
& -(q+p z) \overline{K_{2}}(\lambda-\lambda z)\left[\mathrm{r} \overline{K_{1}}(\lambda-\lambda z) .(q+p z) \overline{K_{3}}\right. \\
& (\lambda-\lambda z)\left[\mathrm{r}^{2} \overline{\mathrm{K}_{1}}(\lambda-\lambda \mathrm{z}) \overline{K_{2}}(\lambda-\lambda z)\right. \\
& -\cdots .-(q+p z) \sum_{j=1}^{n} r^{j-1} \overline{K_{J}}(\lambda-\lambda z) \\
& \mathrm{z}=1, \mathrm{P}_{\mathrm{q}}(\mathrm{z}) \text { in equation }(41) \text { is indeterminate of the form } 0 / 0 .
\end{aligned}
$$


Applying L' Hopital's rule and using $\bar{B}_{l}(0)=$ $1, \bar{B}_{J}{ }^{\prime}(0)=E\left(v_{j}\right), \bar{B}_{J}{ }^{\prime \prime}(0)=E\left(v_{j}^{2}\right), j=1,2,3 \ldots n$

Where $E\left(v_{i}\right)$ is the expected service time for $i$ th service $\mathrm{E}\left(\mathrm{v}_{\mathrm{i}}{ }^{2}\right)$ is the second moment of the service time for the $\mathrm{i}$ th service

We have

$=\lim _{z \rightarrow 1} P_{q}(\mathrm{z})$

$$
=-\frac{\lambda \sum_{j=0}^{n} r^{j} E\left(V_{j+1}\right)}{q-r^{2}\left(p+\lambda E\left(v_{1}\right)+\lambda E\left(v_{2}\right)-\cdots \ldots \ldots-r^{n-1}\left(p+\lambda \sum_{j=1}^{n} E\left(v_{j}\right)\right.\right.} \mathrm{V}(1)
$$

Next, we must have $\mathrm{P}_{\mathrm{q}}(\mathrm{z})+\mathrm{V}(1)=1$

Adding $\mathrm{V}(1)$ to $\mathrm{P}_{\mathrm{q}}(\mathrm{z})$ and equating to 1 , on simplification We get $\mathrm{V}(1)$

$=1-\frac{\lambda \sum_{j=0}^{n} r^{j} E\left(V_{j+1}\right)}{q-r^{2} p-r \lambda\left(E\left(v_{2}\right)+r E\left(v_{1}\right)-\cdots \ldots \ldots-r^{n} \lambda \sum_{j=1}^{n} E\left(v_{j}\right)\right.}$

$\mathrm{V}(1)$ is the steady state probability that the server is under vacation .

Utilization factor is given by $\rho=1-V(1)$

$$
=\frac{\lambda \sum_{j=0}^{n} r^{j} E\left(V_{j+1}\right)}{q-r^{2} p-r^{2} \lambda\left(E\left(v_{1}\right)+E\left(v_{2}\right)\right)-\cdots \ldots \ldots-r^{n} \lambda \sum_{j=1}^{n} E\left(v_{j}\right)} j=1 \text { ton }
$$

\section{MEAN NUMBER IN THE SYSTEM}

Let $\mathrm{L}_{\mathrm{q}}$ denote the mean number of customers in the queue.

From eq (41)

$$
L_{q}=\frac{d}{d z} P_{q}(z) \text { at } z=1
$$

Since $\mathrm{P}_{\mathrm{q}}(\mathrm{z})$ in eq (41) takes the form $0 / 0$, using the result in queueing theory (kashyap and chaudhry)

$$
\begin{aligned}
& \text { We get } \begin{array}{r}
L_{q}=\lim _{z \rightarrow 1} \frac{d}{d z} P_{q}(z) \\
\quad=P_{q}^{\prime}(1)
\end{array} \\
& \lim _{z \rightarrow 1} \frac{D^{\prime}(1) N^{\prime \prime}(1)-N^{\prime}(1) D^{\prime \prime}(1)}{2\left(D^{\prime}(1)\right)^{2}} \\
& D^{\prime}(1)=r \lambda E\left(v_{2}\right)+r^{2}\left[p+\lambda E\left(v_{1}\right)+\lambda E\left(v_{2}\right)+\lambda E\left(v_{3}\right)\right]+ \\
& q \lambda E\left(v_{1}\right)+\lambda E\left(v_{1}\right)+r^{n-1}\left[\lambda E\left(v_{1}\right)+\cdots \ldots .+\lambda E\left(v_{n}\right)-p\right]
\end{aligned}
$$

$$
\begin{aligned}
& D^{\prime \prime}(1)=-r^{2} \lambda^{2}\left[\left[E\left(v_{1}^{2}\right)+E\left(v_{2}^{2}\right)+E\left(v_{3}^{2}\right)\right]+2\left[E\left(v_{1}\right) E\left(v_{2}\right)+\right.\right. \\
& \left.\left.\quad+2 E\left(v_{2}\right) E\left(v_{3}\right)+2 E\left(v_{3}\right) E\left(v_{1}\right)\right]\right]+\cdots \ldots- \\
& \quad r^{n-1} \lambda^{n-1}\left[E\left(v_{1}^{2}\right)+E\left(v_{2}^{2}\right)+\cdots .+2 E\left(v_{1}\right) E\left(v_{2}\right)\right. \\
& \left.\quad+2 E\left(v_{n-1}\right) E\left(v_{n}\right)\right] \_ \text {(45) } \\
& N^{\prime}(1)=\lambda \sum_{j=0}^{n} r^{j} E\left(v_{j+1}\right)
\end{aligned}
$$

$$
\times \frac{\lambda \sum_{j=0}^{n} r^{j} E\left(v_{j+1}\right)}{q-r^{2} p-r^{2} \lambda\left(E\left(v_{2}\right)+E\left(v_{1}\right)\right)-\cdots \ldots . .-r^{n} \lambda \sum_{j=1}^{n} E\left(v_{j}\right)}
$$

$$
\begin{aligned}
& N^{\prime \prime}(1)=\left\{-\lambda^{2}\left[-r^{2} E\left(v_{1}^{2}\right)+E\left(v_{1}^{2}\right)+r E\left(v_{2}^{2}\right)-r^{2} E\left(v_{3}^{2}\right)\right.\right. \\
& \left.\quad+\ldots \ldots \ldots+r^{n} E\left(v_{n}^{2}\right)+2 r^{n} E\left(v_{1}\right) E\left(v_{n}\right)\right]
\end{aligned}
$$

$\times \frac{\lambda \sum_{j=0}^{n} r^{j} E\left(V_{j+1}\right)}{q-r^{2} p-r^{2} \lambda 9\left(E\left(v_{2}\right)+E\left(v_{1}\right)\right)-\cdots \ldots \ldots-r^{n} \lambda \sum_{j=1}^{n} E\left(v_{j}\right)}$

Substituting the values (44),(45),(46) and (47) in (43), we get $\mathrm{L}_{\mathrm{q}}$ in closed form. Further using Little's formula, the average system size $\mathrm{L}$ is calculated.

From the equations (42) and (43),

we have $L=L_{q}+\rho$

\section{MEAN WAITING TIME}

The mean waiting time in the queue and in the system are obtained using Little's formula.

$$
\begin{gathered}
W_{q}=\frac{L_{q}}{\lambda} \\
W=\frac{L}{\lambda}
\end{gathered}
$$

Where $L_{q}$ and $L$ have been found in equations

(43)and (48)

\section{CONCLUSION}

This paper clearly analyses a multi phase M/G/1 feedback queue with multiple server vacation. This service is advantageous to the customers. First service is essential, other services are optional. If the customer is not satisfied with the service, again he can join the tail of the queue and get the regular service. This type of service can be widely used in production industries system, bank services, computer and communication networks. This type of service will give a complete satisfaction to the customers.

\section{REFERENCES}

[1] Disney, R.L., Mcnickle, C.D., and Simon, B. 1980. The M/G/1 queue with instantaneous Bernoulli feedback. Naval Research Logist Quart. 27:635-644.

[2] Disney, R.L. 1981. A note on sojourn times in M/G/1 queue with instantaneous Bernoulli feedback.Naval Research Logist Quart. 28:679-684.

[3] Takagi, H. 1991. A Foundation of Performance Evaluation. Vol. 1. Vacation and Priority systems, Part $\mathbf{I}$ : Elsevier Science, New York.

[4] Kleinrock, L. 1975. Queueing Systems. Vol. 1. John Wiley, New York.

[5] Choi, B.D., Kim, B., and Choi, S.H. 2003. An M/G/1 queue with multiple types of feedback, gated vacations and FCFS policy. Queueing Systems30:1289-1309. 
[6] Boxma, O.J., and Yechiali, U. 1997. An M/G/1 queue with multiple type of feedback and gated vacations. J. Appl. Prob. 34:773-784.

[7] Bertsimas, D., and Papaconstanantinou, X. 1988. On the steady state solution of the M/C2_a_ b_/S queueing system. Transportation Sciences22:125-138.

[8] Madan, K.C. 1992. An M/G/1 queueing system with compulsory server vacations. Trabajos de Investigacion $7: 105-115$

[9] Madan, K.C. 2000. An M/G/1 queue with second optional service. Queueing Systems 34:37-46.

[10] Choudhury, G. 2002. A batch arrival queue with a vacation time under single vacation policy. Computers and Operations Research 29(14):1941-1955.

[11] Choudhury, G. 2003. Some aspects of an M/G/1 queueing system with second optional service. TOP 11(1):141-150.

[12] Choudhury, G., and Paul, M. 2005. A two phase queueing system with Bernoulli feedback. Information and Management Sciences 16(1):773-784.

[13] Kalyanaraman, R., and Pazhani Bala Murugan, S. 2008. A single server queue with additional optional service in batches and server vacation.Applied Mathematical Sciences 2:2765-2776.

[14] Krishna Kumar, B., Vijayakumar, A., and Arivudainambi, D. 2002.An M/G/1 retrial queueing system with two phase service and preemptiveresume. Ann. Oper. Res. 113:61-79.

[15] Medhi, J. 2002. A single server poisson input queue with a second optional service. Queueing Systems 42:239-242.
[16] Scholl, M., and Kleinrock, L. 1983. On the M/G/1 queue with rest periods and and certain service three independent queueing disciplines. Oper. Res.31(4):705-719.

[17] Doshi, B.T. 1986. Queueing systems with vacation-a survey. QueueingSystems 1:29-66.

[18] Keilson, J., and Servi, L.D. 1986. Oscillating random walk models for GI/G/1vacation systems with Bernoulli schedules. J. Appl. Prob. 23:790-802.

[19] Shanthikumar, J.G. 1988. On the stochastic decomposition in the M/G/1 types queues with generalised server vacations. Oper. Res. 36:566-569.

[20] Cramer, M. 1989. Stationary distributions in a queueing system with vacation times and limited service. Queueing Systems 4:57-68.

[21] Heyman, D.P. 1980. Optical operating polices for M/G/1 queueing systems. Oper. Res. 16:362-382.

[22] Lee, T.T. 1984. M/G/1/N queue with vacation time and exhaustive service discipline. Oper. Res. 32:774-784.

[23] Doshi, B.T. 1985. A note on the stochastic decomposition in a $\mathrm{G} / \mathrm{G} / 1$ queue with vacation or set up times. J. Appl. Prob. 22:419-428.

[24] Fuhrmann, S.W., and Cooper, R.B. 1985. Stochastic decompositions in an M/G/1 queue with generalised vacations. Oper. Res. 33:1117-1129. 